\title{
OLIVIER GUYOTJEANNIN
}

\section{RETOUR SUR LE CARTULAIRE BLANC DE SAINT-DENIS}

Le rapide wretour sur le Cartulaire blanc« que je viens proposer, cédant à l'amicale insistance de Rolf Grosse, va considérablement éloigner le lecteur des $\mathrm{XI}^{\mathrm{e}}-\mathrm{XII}^{\mathrm{e}}$ siècles fort opportunément mis au programme d'une journée d'études sur Saint-Denis. Non seulement parce que les deux monumentaux volumes du Cartulaire blanc sont œuvre du dernier quart du XIII ${ }^{\mathrm{e}}$ siècle; mais encore parce que, outil de gestion autant que de mémoire, le Cartulaire blanc survalorise la production elle-même surabondante du demi-siècle qui précède le lancement de l'entreprise: des 2616 actes que j'y ai comptés (avec quelques doublons et non sans risque d'erreurs), une grosse moitié (51\%) est datée des années 1226 à 1275. Le »retour « sera donc détour; pris de remords, j'esquisserai un rapide retour sur Suger en fin d'exposé. Auparavant, je ne ferai plus que livrer quelques informations et éléments de réflexion, tirés d'un travail mené collectivement par les étudiants de l'École des chartes - travail qui n'en est qu'aux premiers pas, ce qui je l'espère fera pardonner le caractère à la fois partiel et temporaire des conclusions.

\section{L'art du cartulaire à Saint-Denis}

Depuis que Rolf Grosse a débrouillé la chronologie des entreprises de compilation de cartulaires à Saint-Denis ${ }^{1}$, on perçoit mieux les voies choisies par l'abbaye pour investir, tard mais pas forcément en retard, et massivement mais pas forcément exhaustivement, dans les entreprises de compilation. L'analyse partielle à laquelle nous avons déjà pu nous livrer corrobore entièrement les conclusions présentées par Rolf Grosse en 1991: de "vrais« cartulaires, il n'en reste que du XIII ${ }^{\mathrm{e}}$ siècle.

1 Rolf GROSSE, Remarques sur les cartulaires de Saint-Denis aux $\mathrm{XIII}^{e}$ et $\mathrm{XIV}^{\mathrm{e}}$ siècles, dans: Olivier GUYOTJEANNIN, Laurent MORELLE, Michel PARISSE (dir.), Les cartulaires, actes de la table ronde organisée par l'École nationale des chartes et le G.D.R. 121 du C.N.R.S., Paris 5-7 décembre 1991, Paris, Genève 1993 (Mémoires et documents de l'École des chartes, 39), p. 279-288. 
Une première vague, hétérogène, produit d'abord des cartulaires spécialisés, de faible volume. D'une part avec un cartulaire du convent, compilé vers la fin des années 1220 , riche par définition en actes des abbés, et presque exclusivement en actes des quatre dernières décennies; dit à tort cartulaire »de l'Aumônerie«, il est repris un demi-siècle plus tard; le convent n'ayant décidément pas de chance, ce cartulaire-ci sera dit »de la Pitancerie«, et assez vite, peut-être dès l'origine, il est venu former comme un appendice du Cartulaire blanc. L'autre cartulaire spécialisé, lui, mérite mieux son nom de »Livre des privilèges«, et moins son sort, puisqu'il est aujourd'hui largement illisible; il compile bulles et diplômes, sans omettre ni la pseudo-Donation de Constantin ni un acte de Suger (enfin!), dans les décennies centrales du XIII ${ }^{\mathrm{e}}$ siècle. Je rejoins sans réticence la conclusion de Rolf Grosse: le plan de classement laisse ouverte la possibilité que le volume actuel procède de la reprise et de la mise à jour d'un cartulaire d'»autorités«, compilé vers le milieu du XII ${ }^{\mathfrak{e}}$ siècle, et pourquoi pas à la fin de l'abbatiat de Suger ou peu après - ce serait la seule trace tant soit peu sûre d'un cartulaire du XII ${ }^{\mathrm{e}}$ siècle.

Après ces premiers essais, deux autres vagues viennent manifester des projets autrement ambitieux. On peut dater des années 1277-1278 la fin de l'étape primitive de compilation du Cartulaire blanc, marquée par la réalisation d'une table récapitulative qui servira encore au début du XIV siècle d'outil d'accès, bien incommode, aux originaux du chartrier. Jusqu'en 1300, la mise à jour du Cartulaire est assez systématique; on n'y transcrit plus ensuite que quatre actes. Le relais est pris, autour des années 1320, par un projet aussi ambitieux mais inachevé, plus technicien et plus brouillon, de cartulaires spécialisés par domaines, reprenant une documentation récolée, cotée et inventoriée à nouveaux frais.

La masse de la documentation engrangée à Saint-Denis a parallèlement stimulé les brillants archivistes de l'abbaye, qui ont déployé une rare ardeur à annoter, marquer, ranger, inventorier leur chartrier. L'importance de l'atelier historiographique san-dionysien n'est peut-être pas étrangère à la qualité de leurs travaux; elle ne l'est sûrement pas à la valorisation de leur fonction et à la conservation méticuleuse d'un ensemble d'inventaires d'archives, exceptionnel par sa densité - l'autre facteur d'explication à cette conservation étant que les finalités de chacun ayant considérablement varié, ils gardaient tous une valeur propre qui incitait à ne pas les détruire ${ }^{2}$.

2 Pour plus de détail, mais sans aucune exhaustivité, Olivier GUYOTJEANNIN, La science des archives à Saint-Denis, fin du XIII ${ }^{e}$-début du $\mathrm{XVI}^{e}$ siècle, dans: Françoise AUTRAND, Claude GAUVARD, Jean-Marie MOEGLIN (dir.), Saint-Denis et la royauté, études offertes à Bernard Guenée, Paris 1999 (Histoire ancienne et médiévale, 59), p. 339-353, et La tradition de l'ombre: les actes sous le regard des archivistes médiévaux (Saint-Denis, XII $-\mathrm{XV}^{e}$ siècles), dans: Adam J. KOSTO, Anders WINROTH (dir.), Charters, cartularies and archives: the preservation and transmission of documents in the medieval West, proceedings of a colloquium of the Commission internationale de diploma- 
Non seulement le Cartulaire blanc est contemporain de l'édition officielle (1274) du premier noyau de ce qui va devenir les Grandes chroniques de France, pour lesquelles Primat prend plus d'une fois le chemin du chartrier; mais encore son confrère et collègue Guillaume de Nangis, officiellement en charge des archives et responsable de la mise à jour du Cartulaire, ne peut être que l'instigateur plus ou moins proche d'une cuvre parfaitement originale, un »digest« résumant le Cartulaire et compilé d'une traite entre 1287 et 1289 . Un obscur destin a valu à cette compilation le nom d'»Ancien inventaire noir", bien à tort car il n'est plus noir et n'a rien d'un inventaire d'archives. Depuis quelques années, je ne cesse de m'émerveiller devant ce petit volume qui montre comment les historiens-archivistes de Saint-Denis lisaient, glosaient et parfois déformaient le texte de leurs actes anciens et moins anciens.

La confection de l'»Ancien inventaire noir« est assurément l'élément le plus original dans le dispositif de compilation du Cartulaire blanc. Car, pour le reste, le Cartulaire blanc brille moins par l'originalité de sa facture que par sa masse. Ni sa table, clef d'accès aux originaux, ni ses mises à jour programmées sur un quart de siècle, ni ses rubriques, ni ses transcriptions exhaustives et soignées (du latin au moins, car la transcription de la langue vernaculaire est rien moins que littérale, mais cela tient plus à l'état de la langue qu'aux capacités du compilateur), agrémentées de quelques renvois directs au chartrier et aux actes répétitifs qu'il renferme, ni son ornementation sobre, ni la multiplication des outils fonctionnels de repérage ne dénoncent beaucoup d'innovations. Le plan, un peu perturbé à la reliure, n'est pas plus original, qui donne l'avantage à la répartition des chapitres par domaines ou ensembles géographiques, çà et là regroupés ou identifiés à contretemps (soixante-quatre chapitres "topographiques«, contre quatre consacrés à des offices et quatre encore à des types d'actes ou d'objets). Quelles particularités signaler alors? Serait-ce la présence constante du dessin des monogrammes royaux? Elle retient surtout l'attention quand des bévues (le monogramme de saint Louis pour celui de Louis VI) montrent que l'illustrateur, comme Primat résumant des diplômes de Robert le Pieux dans les Grandes chroniques, travaille avec des fiches qu'il mélange parfois. Seraient-ce les incohérences troublantes de numérotation qui, entre le corps du Cartulaire (où elle est assez tardive), sa table et l'Ancien inventaire noir, amènent parfois à décerner trois numéros différents au même acte, tous distincts du numéro porté au dos de l'original? On en conclut surtout que le cartulaire fait écran devant la chartrier, autrement dit qu'il sert à quelque chose ...

Le plus intéressant dans la fabrication du volume vient peut-être du mode de classement des actes à l'intérieur des chapitres. Le chapitre initial, "SaintDenis«, qui mélange les actes ponctuels sur la localité et les actes généraux 
(royaux et abbatiaux, puisque les bulles sont à part), est le seul dont le plan, par catégories d'auteurs, reprenne, en partie, celui du Livre des privilèges. Partout ailleurs, un primat évident est donné au classement chronologique. Et ce qui frappe, quand le travail a été poussé jusqu'au bout, c'est le savoir-faire et l'opiniâtreté appliqués presque sans faille à l'exécution: savoir-faire qui permet aux archivistes d'attribuer, presque toujours à bon escient, les diplômes carolingiens, datés de la seule année de règne, au bon Charles; opiniâtreté qui, dans les cas les plus aboutis, les amène à classer les documents non seulement par année mais encore par mois et parfois par jour, et sans se laisser abuser par l'ancien style.

Autre intérêt du classement chronologique: il est le fil d'Ariane pour dégager un peu mieux les étapes du travail. De-ci de-là, en effet, l'ordre chronologique, très strictement respecté jusqu'aux documents des années 1244-1246, est ensuite rompu - une rupture parfois accusée, au tome II, par de subtils changements graphiques: passé ces années, l'ordre chronologique est volontiers tourmenté, la succession des actes est entrelardée de paquets de munimi$n a$, brisée de retours en arrière, quand elle n'est pas ouverte par un ou deux préceptes carolingiens omis jusque-là. En l'état actuel de l'analyse, bien imparfait, il semble que les compilateurs du Cartulaire blanc, qui ont dû se mettre au travail assez tôt dans les années 1270 , ont d'abord eu à leur disposition des layettes dont un nombre appréciable (surtout pour les possessions les plus proches de l'abbaye) avaient été méticuleusement classées au milieu des années 1240; la suite aurait été mise en ordre (par les compilateurs ou par leurs prédécesseurs immédiats) avec une certaine hâte mais avec des effets impressionnants, comme si l'on avait voulu ratisser large dans un chartrier à moitié classé, réintégrant des actes parfois anciens d'un voire de plusieurs siècles et offrant une documentation beaucoup plus étoffée en même temps que scrupuleusement tenue à jour.

Constatation qui amène à poser l'autre grande question: celle de la richesse du cartulaire. Je l'ai dit en commençant: un peu plus de la moitié des actes transcrits au Cartulaire blanc sont du seul demi-siècle qui, en gros, précède le lancement de la compilation. Le chiffre inquiète, même si l'on tient compte de la courbe générale d'évolution de la production documentaire, qui se confirme d'ailleurs pleinement à Saint-Denis. Dans l'ensemble du Cartulaire blanc, la part des actes antérieurs à 1200 (et même en y incluant une frange d'actes sans date qui pourraient être aussi bien du début du XIII ${ }^{\mathfrak{e}}$ siècle) est de moins d'un cinquième $(18 \%)^{3} ; 3 \%$ d'actes antérieurs à l'an mil, c'est honorable, mais encore fort peu pour Saint-Denis, où l'on sait que par dizaines les préceptes mérovingiens et carolingiens dormaient dans les layettes des "Vieux préceptes« et des »Vieux échanges«, pour n'être guère dérangés avant le $\mathrm{XIV}^{\mathfrak{e}}$ siècle où ils

3 Mais de près de $30 \%$ dans le seul tome II: phénomène bien connu, les possessions plus lointaines, regroupées dans ce volume, incitent plus tôt à produire plus d'écrit. 
seront cotés, sans être jamais recopiés ni inventoriés (c'est le cas d'un original sur trois des nombreux actes donnés à l'abbaye par Charles le Chauve). Quelques sondages grossiers confirment que la sélectivité du Cartulaire blanc est proportionnelle à l'ancienneté des documents; sur la base des actes dont on a encore le texte, tous modes de tradition confondus, le Cartulaire blanc retranscrit à peine plus de la moitié des documents antérieurs à 1150 , mais plus de neuf sur dix des actes de 1225 à $1275^{4}$. Une approche plus fine, sur un autre échantillonnage, confirme que les plus hautes époques sont les premières à faire les frais de cette sélectivité, qui connaît ensuite de légères fluctuations sur le temps court:

\begin{tabular}{|c|c|c|c|c|}
\hline Période & $\begin{array}{c}\text { Originaux, } \\
\text { transcrits au } \\
\text { Cart. blanc }\end{array}$ & $\begin{array}{c}\text { Originaux, } \\
\text { non transcrits au } \\
\text { Cart. blanc }\end{array}$ & $\begin{array}{c}\text { Cart. blanc, } \\
\text { originaux per- } \\
\text { dus }\end{array}$ & $\begin{array}{c}\text { Taux de cou- } \\
\text { verture du Cart. } \\
\text { blanc }\end{array}$ \\
\hline $\mathrm{IX}^{\mathrm{e}}-\mathrm{X}^{\mathrm{e}}$ siècles & 23 & 43 & 11 & $44 \%$ \\
\hline $1101-1150$ & 49 & 9 & 30 & $90 \%$ \\
\hline $1216-1220$ & 56 & 22 & 48 & $83 \%$ \\
\hline $1251-1255$ & 44 & 21 & 28 & $77 \%$ \\
\hline
\end{tabular}

\section{Un projet d'édition}

En dépit de ce déséquilibre, la documentation engrangée reste écrasante et, si sa masse n'a pas découragé les archivistes monastiques, elle a, faut-il le rappeler, effrayé les éditeurs. Les cartulaires de l'abbaye engrangent sans doute pas loin de 3000 actes antérieurs à 1300 , mais ce n'est pas le seul malheur: les originaux sont conservés à foison (dans la proportion de près de deux actes sur trois pour quelques dossiers déjà exploités) mais dispersés; les copies libres sont innombrables et pas toujours inutiles. Et l'ensemble est encore un peu plus éclaté que le tout-venant des chartriers ecclésiastiques entrés sous la Révolution aux Archives nationales, puisque, sous le règne de Louis XIV, la Maison royale Saint-Louis à Saint-Cyr se vit affecter les revenus de la mense abbatiale et, en conséquence, des lots d'originaux, aujourd'hui conservés aux Archives départementales des Yvelines.

C'est donc sous l'effet double d'une témérité qui commence à me faire trembler, et du besoin pressant de fournir vite beaucoup de travail à l'insatiable appétit des promotions successives de l'École des chartes, dont les étudiants s'étaient joué en deux ans des 224 actes du chartrier des Prémontrés

${ }^{4}$ Comptages réalisés sur la base des originaux encore conservés à la fin du XVII et au début du XVIII ${ }^{e}$ siècle au témoignage de l'exceptionnel Inventaire général lancé en 1680 (Arch. nat., LL 1189-1190). 
de Saint-Yved de Braine, et en deux ans encore des 274 formules du formulaire d'Odart Morchesne, que j'ai espéré trouver un peu de répit en les lançant sur le chartrier de Saint-Denis. J'avais d'abord pensé engager le travail de la façon la plus classique qui fût: partir des originaux, recenser les copies, monter assez vite une édition critique en choisissant des termes chronologiques raisonnables - laisser les hautes époques à leurs spécialistes, laisser aussi de côté un long $\mathrm{XI}^{\mathrm{e}}$ siècle sur lequel une entreprise était annoncée, commencer donc à l'abbatiat de Suger (que revoilà), s'arrêter enfin à 1220, où explose la documentation, pour concorder au plus près avec les entreprises de recensement menées à l'I.R.H.T. par Annie Dufour, qui mettait avec la disponibilité que l'on imagine tous ses dossiers à notre disposition. Prendre, en bref, une tranche d'un siècle, qui allait ravir (peut-être) les spécialistes de l'époque, et une fois encore désoler les autres; et qui surtout allait morceler (et en fait interdire) l'analyse fine des cartulaires et des inventaires d'archives, proscrire toute vue d'ensemble sur la gestion du chartrier, questions qui n'étaient pas pour rien dans le choix de Saint-Denis. Alors que tous et chacun déplorent les lacunes dans la connaissance des sources, difficiles, du XIII ${ }^{\mathrm{e}}$ siècle, ne fallaitil sauter le pas? Pour emporter la décision, il a suffi de lire de façon cursive quelques chapitres de Cartulaire blanc, qui montrèrent aussitôt que les sources difficiles du XIII ${ }^{\mathrm{e}}$ siècle sont aussi enthousiasmantes.

Un seul exemple, je pense, illustrera de façon convaincante ces richesses, exemple que je tire du chapitre préparé l'an dernier, consacré à Tremblay-enFrance, accessoirement à Mitry et à Villepinte, et riche de 51 actes (pour 36 transactions différentes). Dense mais aussi disproportionnée qu'ailleurs (19 actes seulement jusqu'en 1220), la documentation offre une possibilité, il est vrai assez rare par son intensité, de recoupement avec le passage consacré au domaine dans le De administratione de Suger. Surtout, en dehors de l'enchâtellement de la localité, de la liquidation de l'avouerie des comtes de Dammartin, de la démultiplication des habitats et des granges, elle livre un dossier fourni sur les seigneurs de Villepinte, rameau des Bouteillers de Senlis, un paquet de munimina remis par les seigneurs, intéressant eux-mêmes comme un gendre, sergent bien marié de Philippe Auguste, Roger la Pie, concernant Paris aussi bien qu'Étampes; en bref autant d'occasions de voir l'implantation de fidèles du Capétien qui a longtemps chassé sur ces terres; et encore d'autres merveilles, provenant du rayonnement du chartrier monastique, bien au-delà des finalités gestionnaires: un témoin, direct et indirect, de la pratique de rédaction par l'abbé d'actes de douaire au profit d'habitants (1206); le témoignage sur la conservation (incertaine au reste) par des laïques d'un contrat de concession de terre à part de fruits (1217-1218); plus tard dans le siècle, trois actes fort précis sur une exécution testamentaire et la création d'une chapellenie. 
Autre extraordinaire occasion, cette fois proprement diplomatique, celle qui est offerte, par la densité même des actes, de découvrir la vie des formules aux années 1230-1270, formules qu'il faut ne pas avoir lues pour les taxer de stéréotype, alors que la rédaction, pour être toujours plus savante, n'a rien de figé. Sur un point particulier, le dossier donne à toucher du doigt le progressif affermissement de la maîtrise rédactionnelle des lettres de Châtelet qui, d'abord engoncées dans le malhabile démarquage du latin de l'officialité épiscopale, au risque permanent du contresens, gagnent en une quinzaine d'années la maturité de leur expression française, offrant aussi à la lexicographie juridique de formidables attestations.

\section{Une édition électronique}

Bref, la tentation l'a emporté, et notre angle d'attaque du chartrier de SaintDenis est donc, désormais, le matériau, tout le matériau du Cartulaire blanc, édité dans l'ordre de la compilation, et pour l'heure en suivant sa transcription. S'il devait rester définitif, le parti serait intenable, puisque le Cartulaire est sélectif, et qu'il pèche, comme tous les copistes. Pour compenser le double péril de cette conception, assumé mais réel, revendiqué comme provisoire, nous recherchons dès maintenant les originaux conservés et les éditions disponibles (très largement limitées aux actes royaux et pontificaux), comme nous pistons dans les divers inventaires d'archives (Ancien inventaire jaune, Inventaire de 1520 , Inventaire de 1680 ) le bilan de la conservation des parchemins. Mais, dans cette première étape, le texte des originaux n'est sollicité que lorsque le texte du Cartulaire soulève un doute à la lecture - la seule excuse reposant dans les tests qui ont montré une large fiabilité du Cartulaire. Par contre, l'intérêt pour les pratiques archivistiques nous incite, dès cette première étape, à relever et éditer non seulement les rubriques du Cartulaire, mais encore le texte des regestes médiévaux (Ancien inventaire noir et Ancien inventaire jaune).

S'il y a une justification à des pratiques aussi barbares, elle est dans la conjonction d'un chiffre et d'un outil. Le chiffre, c'est bien sûr celui de la documentation disponible: au début 2003, le chapitre Tremblay est entièrement traité, le chapitre Rueil [de Rueil-Malmaison] en est au stade du finissage, sans compter la préparation du chapitre Beaurain et quelques sondages dans les chapitres Cergy et Cormeilles. En bref, au rythme actuel, occupant les étudiants de deuxième année, la première phase du travail (édition »soignée« du seul Cartulaire blanc) sera achevée en 2045; la collation des originaux et des autres copies, l'exploitation des autres cartulaires devraient mener tranquille- 
ment l'entreprise à l'orée du XXII ${ }^{\mathfrak{e}}$ siècle. Voilà pour le chiffre. L'outil qui sauve l'honneur, c'est l'édition électronique dont le webmestre du site de l'École des chartes, Gautier Poupeau, affine la conception depuis le début de $2002^{5}$. Outre la volonté d'explorer les potentialités, encore mal reconnues, d'une édition informatique de documents diplomatiques, ce choix était presque imposé par le »dossier et son mode de traitement: souci de fournir dès maintenant des éléments, utilisables et modifiables à la fois, sans attendre un siècle; possibilité de donner à lire une masse de textes du second $\mathrm{XIII}^{\mathrm{e}}$ siècle, que la longueur du formulaire exclut, par la logique économique, de l'édition papier.

Pour le dire rapidement, la lecture des actes édités peut se faire par de multiples accès: $1^{\circ}$ à partir de l'ordre du Cartulaire (une introduction étoffée détaillant, pour chaque chapitre, l'historique du domaine, la composition du chapitre, mais comprenant aussi un volet iconographique aussi diversifié que possible: cartes de localisation, documents graphiques anciens, tableaux généalogiques, photographies ..., et un volet pédagogique, avec des suggestions d'utilisation des actes); $2^{\circ}$ à partir d'une table chronologique cumulative, qui semble à peu près au point; $3^{\circ}$ à partir de divers échelons de cartes visualisant soit les domaines, soit les quartiers de chaque finage; $4^{\circ}$ enfin, c'est le plus classique et le plus difficile à mettre en ouvre, à partir d'index, où - est-il besoin de le dire? - nous buttons sur les problèmes de masse, de renvois, de lemmatisation. Il n'est guère plus besoin de dire que la construction progressive de l'édition, la mise à jour des index et des tables, l'enrichissement ultérieur des collations sont, de leur côté, des problèmes automatiquement résolus. Le principe de l'association des textes (ou de leur table pour les chapitres non encore édités) à une numérisation intégrale du cartulaire est également retenu, en partenariat avec les Archives nationales. J'irai vite sur la discussion des choix fondamentaux liés à l'édition informatique, pour livrer deux éléments: d'une part, nous entendons maintenir fermement le principe de la gratuité de consultation (diverses procédures facilitant même l'impression); d'autre part, le danger d'une atomisation de la consultation est combattu par des outils qui tendent toujours à remettre l'information en contexte (introductions, tables récapitulatives de divers types, cartes de position ...), domaine où tout reste à inventer!

5 Les premiers éléments disponibles (pages et cartes d'orientation générale, tableau des chapitres, chapitre Tremblay entièrement traité) sont consultables sur le site de l'École des chartes (www.enc.sorbonne.fr). Une présentation plus développée de la conception du site, par O. GUYOTJEANNIN et Gautier POUPEAU, a parue dans: Le médiéviste et l'ordinateur 42 (2003), p. 93-99. L'architecture informatique a notablement évoluée depuis cette mise au point. 


\section{Retour sur Suger}

En attendant de voir Suger surfer, cette rapide présentation doit se clore sur une question où sa figure rencontre le Cartulaire blanc, question qui, à ma connaissance, n'a jamais été abordée par les éditeurs des chartes de Suger: quelle a été à l'abbaye la postérité et la célébrité des actes de l'abbé? Les a-ton lus et copiés? Comment les a-t-on appréciés et compris? Questions intéressantes, je pense, mais auxquelles l'histoire archivistique n'apporte que des lueurs maigres et ambiguës, avec pour seul avantage de susciter quelques interrogations.

Des seize actes intitulés au nom de Suger qui nous sont aujourd'hui connus, cinq viennent d'autres chartriers ou, à tout le moins, n'ont pas intégré le chartrier de Saint-Denis avant la fin du Moyen Âge ${ }^{6}$ : un acte pour Saint-Martindes-Champs $\left(n^{\circ} 14\right)$, un autre pour Valséry $\left(n^{\circ} 15\right)$, un acte pour le chapitre Saint-Paul de Saint-Denis $\left(\mathrm{n}^{\circ} 6\right)$, selon toute évidence d'abord conservé hors de l'abbaye ${ }^{7}$, deux actes enfin pour le prieuré san-dionysien de La Chapelaude dont toute la tradition est issue du prieuré et non de l'abbaye-mère ( ${ }^{\text {os }} 4$ et 7$)$. Mais onze actes (un chiffre considérable encore que non exceptionnel pour des bénédictins) se sont retrouvés au chartrier du producteur (annexe 2): certains parce qu'ils sont délivrés en winterne « et concernent des affectations à des offices, des fondations de messes et d'anniversaires ..., et Suger en a été prodigue, d'autre parce qu'ils relèvent d'une grande et assez ordinaire prudence de gestion, l'un des exemplaires ou le seul exemplaire étant archivé à l'abbaye ${ }^{8}$.

Où les choses deviennent plus dignes de remarque, c'est que, sur ces onze actes, neuf sont transcrits, un siècle et demi après, au Cartulaire blanc, dont cinq en bloc, au premier chapitre, "Saint-Denis« - seule occasion de sauver quatre actes qui ont vu leur original disparaître. Occasion aussi de voir com-

6 Référence est faite désormais aux numéros de la section »Chartes« de la dernière édition en date: Suger, Euvres, t. 2: Lettres de Suger, Chartes de Suger, Vie de Suger par le moine Guillaume, texte établi, traduit et commenté par Françoise GASPARRI, Paris 2001 (Les classiques de l'histoire de France au Moyen Âge, 41), p. 155-267.

7 À la différence du grand acte sur les rapports entre l'abbaye et le chapitre $\left(\mathrm{n}^{\circ} 11\right)$, celui-ci ne semble pas avoir intégré le chartrier abbatial avant quelque temps; l'original (Arch. nat., $\mathrm{L} 845, \mathrm{n}^{\circ} 19$ ) porte au dos une analyse de la fin du XIII ${ }^{\mathrm{e}}$ siècle qui differe des analyses usuellement portées à l'abbaye à l'époque (Littere bone memorie Suggerii quondam abbatis Sancti Dyonisii de molendino defuncti Yvonis, de compositione facta inter cantorem et capitulum Sancti Pauli et Adam filium defuncti Yvonis anno Domini millesimo $\left.C^{\circ} X X X^{\circ} V^{\circ}\right)$. La cote dorsale (? XV siècle: $\left.+a a R\right)$ est le premier indice d'une conservation au chartrier de l'abbaye de Saint-Denis.

8 Le cas est évident pour le $n^{\circ} 16$, établi sous la forme d'un chirographe, dont un exemplaire est transcrit au Cartulaire blanc de Saint-Denis, et dont l'autre, conservé en original aux Archives nationales ( $\mathrm{L} 1004, \mathrm{n}^{\circ} 2$ ), vient du chartrier de Longpont au témoignage de ses notes dorsales. 
ment Guillaume de Nangis ou son aide comprennent et transmettent la substance de ces actes, essentiellement des cinq premiers, puisqu'une partie de l'Ancien inventaire noir est manquante (annexe 1): je relève, pêle-mêle, le souci discret mais effectif d'attirer l'attention sur la personne de l'abbé (que parallèlement Primat mettait en relief dans ses Grandes chroniques ${ }^{9}$ ), par des initiales tant soit peu décorées, une pratique exceptionnelle dans ce petit volume pragmatique; l'expertise juridique et lexicale; le souci de gloser aussi (l'analyse ajoutant ici des précisions d'ordre liturgique, là la localisation de la sépulture de l'abbé); la déformation enfin qu'impose le souci gestionnaire: une longueur inusuelle est réservée au regeste (qui se fait, par moments, transcription pure et simple) de l'acte fixant le statut du chapitre Saint-Paul, pièce de choix apparemment pour décrire les relations avec l'abbaye.

À ces transcriptions, pourtant, il manque, et ils sont les seuls à manquer, les deux plus beaux fleurons sugériens du chartrier: les deux testamentsordonnances de 1137 et 1140, qui fascinent les historiens mais vivent aux archives d'une vie presque souterraine, soulevant bien des questions sur ce que nous pouvons appréhender des chartriers médiévaux, même quand on aura dit que le Cartulaire blanc a dû les négliger parce qu'ils ne regardent pas assez la gestion et trop le convent - mais ils ne sont pas plus aux cartulaires du convent, et le Cartulaire blanc transcrit bien la fondation de l'office de la Vierge, qui n'est pas le seul à augmenter les distributions! Le premier »testament«, de 1137, a certes été transcrit au Livre des privilèges; il porte une analyse dorsale du XII ${ }^{e}$ siècle (De anniversario abbatis Sugerii), et un numéro d'ordre du XIV', qui permet de le rapprocher d'une analyse de l'Ancien inventaire jaune ${ }^{10}$. Il était donc bien aux archives »centrales« de Saint-Denis, ce qui confirme qu'il a été délibérément écarté lors de la préparation du Cartulaire blanc. Plus troublant encore, le cas du second stestament«, de 1140: pourtant composé selon toute vraisemblance quelques années après lui (après 1146), le prototype du Livre des privilèges l'ignore, au profit du seul acte de 1137. Mais nous conservons encore son original, notre unique source, un original qui ne surgit à nos yeux qu'avec l'inventaire de 1520; avant la cote qui est inscrite sur son dos à la fin du $\mathrm{XV}^{\mathrm{e}}$ siècle, l'original ne porte aucune de ces annotations qui constellent le dos de presque tous les autres actes: pas d'analyse du XII ${ }^{e}$ et du XIII ${ }^{e}$ siècle, ni de »cotes« des $\mathrm{XIII}^{\mathrm{e}}$ et $\mathrm{XIV}^{\mathrm{e}}$. Aurait-il

Voir la glose de Primat, insérée dans un passage traduit de Suger: "Moines [on en est encore au règne de Philippe $F^{e}$ ] estoit de Saint Denys en France et puis fu il abbés de laienz et fit tant de biens ou roiaume et a l'Eglise, car il ot tot le roiaume en sa garde au tens que lui rois Loois, fiuz Loois le Groz et peres au roi Philippe, fu outre mer. Et si fu cil maimes qui escrit cete estoire, si certenement comme cil qui ausi, come toz jors, fu norriz ou palais et ou servise le roi«, éd. Jules VIARD, Les Grandes chroniques de France, t. 5, Paris 1928 (Société de l'histoire de France), p. 115.

${ }^{10}$ Sugerii abbatis quomodo constitutum fuit anniversarium suum in capitulo, cum isto signo $+a b$ : Arch. nat., LL 1185, p. 52. 
entièrement vécu dans l'ombre du testament de 1137 (dont il imite par ailleurs la présentation matérielle), avec qui il partage en 1520 une même cote et une même analyse ${ }^{11}$, que l'on ne s'expliquerait ni le silence du Livre des privilèges ni celui de l'Ancien inventaire jaune.

La question est ainsi repoussée dans le camp des historiens du XII ${ }^{e}$ siècle san-dionysien, où je me suis imprudemment avancé avant de m'en retourner au Cartulaire blanc.

\section{Annexe 1}

\section{Analyses d'actes de Suger aux années 1280}

On édite ici les regestes donnés par l'Ancien inventaire noir (Arch. nat., LL 1184), autrement dit par Guillaume de Nangis ou sous sa direction, d'actes transcrits dans le Cartulaire blanc. En raison de lacunes du volume, seuls sont disponibles les regestes des actes transcrits aux chapitres Saint-Denis et Lavercines. On mentionne en tête le numéro de la charte dans l'édition Gasparri, puis la pagination du regeste dans l'»Inventaire«. On signale par un interlettrage les principales adjonctions faites par l'»Inventaire« au texte des actes.

[Charte $n^{\circ} 5=$ p. 11] Suggeri abbatis et conventus quomodo concessimus Roberto Aurifabro unam aream ad domum construendam ante monasterium nostrum hac pactione ut redderet annuatim nobis infra octabas Sancti Dyonisii unum coclear argenteum ponderis unius uncie.

[Charte $\mathrm{n}^{0} 1=$ p. 11-12] Suggeri abbatis quomodo ipse constituit fieri omni feria sexta servicium trium lectionum de beata Maria, sicut mos est nobis facere tres lectiones infra octabas Pentecostes tribus penultimis diebus. Et ita precepit fieri omni feria quinta de beato Dyonisio, nisi accidant XII lectiones hiis diebus ${ }^{12}$. Propter istud servicium faciendum ipse augmentavit cotidianum generale nostrum de sex solidis, nam antea IIIf solidorum erat $t^{13}$, et ita debemus habere cotidie $X$ solidos pro generali. Item ipse constituit ut, quocienscumque essemus cenaturi, quod haberemus refectiones de decima Sancti Luciani.

[Charte $\mathrm{n}^{0} 3=$ p. 12] Suggeri abbatis quomodo ipse absolvit comitem Mospecensis (sic) de quibusdam dampnis nobis illatis.

[Charte $\mathrm{n}^{\circ} 2=$ p. 12] Suggeri abbatis et conventus quomodo homines ville Beati Dyonisii fuerunt libertati traditi.

11 »Deux chartres de l'abbé Suggectz touchant son obit et plusieurs droictz par [pour] la fondacion d'iceluy pour le convent tant en refections conventuelles comme aultrement $«$ : Arch. nat., LL 1187, p. 121.

${ }^{12}$ La charte évoque un déplacement dû aux principales solennités de vigiles ou du Carême.

${ }^{13}$ La charte prescrit l'inverse, une augmentation de 4 sous du quotidien auparavant fixé à 6 sous. 
[Charte $\mathrm{n}^{\circ} 11=$ p. 12-13] Suggeri abbatis et conventus quomodo concessimus canonicis Sancti Pauli tenere claustrum suum liberum et immune ab omni exactione et domos et officinas claustri et quas circun (sic) claustrum habere poterunt et ubicumque habuerint domos suas quamdiu in eis habitaverint et res eorum et proprie familie; si autem de manibus eorum ad alias personas devenerint, in potestatem judiciarie potestatis lege ville redibunt. Res opidanorum nostrorum vel aliorum hominum in domibus eorum nulla defendet libertas. Hoc tantum fiet quod res aliorum per clericos Sancti Pauli potestati nostre reddentur. Latrones suos de familia sua et qui eis furati fuerint eorum sit ad justiciandum; fugitivos alios latrones potestati nostre reddent, similiter et alios qui ad eos confugerint reos. Item concessimus eisdem in utraque Sancti Pauli festivitate percipere annuatim apud Duolium XL solidos de censu mansi Arnulphi et modium unum annone in molendino uno apud Ulmeçon atque modium unum vini in celario nostro cum decem solidis de censu vini clamatorum. Item concessimus eisdem ecclesiam Sancti Johannis et medietatem decime ville que dicitur Ablegias et quartam partem decime de Barcheniaco cum medietate decime ville que dicitur Campiniacus. Item concessimus eisdem habere a nobis annuatim in anniversario Suggerii abbatis unum modium vini et centum panes ad mensuram panis nostri de refectorio, eo pacto et ea conventione ut ipsi celebrent annuatim in capitio ecclesie nostre ${ }^{14}$ anniversarium Suggerii abbatis et in obitu singulorum fratrum nostrorum ad corpora nondum sepulta conveniant ibique commendationem anime faciant et ad matorium sancti Hilarii pro eadem missam celebrent; et ita similiter de monachis extra abbatiam defunctis tenentur facere. Item pro decantatione psalterii quam post matutinos nostros se non posse facere testati sunt, statutum est ut postquam frater noster defunctus sepultus fuerit, in crastinum vel infra triduum post sepulturam ejus conveniant in ecclesia Sancti Pauli et restaurationem psalterii commendationemque anime ac missam pro ejus anima pariter decantent. Item dedimus eisdem ecclesiam Sancti Petri pro matutinis decantandis annuatim in vigilia Beati Dyonisii in capitio ecclesie nostre ante nostros matutinos, sicut reperire poteritis in prima carta de titulo Sancti Pauli ${ }^{15}$. Item Robertus rex dedit eis molendinum de Sancto Luciano et de Ponte Maberti, item furnum de Sancto Marcello et de Porta Basuini. Item dedimus clausum de Strata, clausum de Cormeliis, clausum de Monte Morenciaco et clausum de Dyogilo cum quantula census portione que circa villam et infra adjacet.

[Charte $\mathrm{n}^{\circ} 16=$ p. 367] Sugerii abbatis quomodo concessimus monachis Longipontis VI modiatas terre juxta territorium nostrum in Monte de Pois pro duobus modiis, uno mediocris frumenti et altero avene, percipiendis annuatim ad festivitatem Omnium Sanctorum in granchia ipsorum de Valle Beron ad mensuram Suessionis ${ }^{16}$. Item remisimus eisdem in dictis sex modiatis decimam et terragium.

${ }^{14}$ La charte de Suger portait: in die anniversarii mei, si fidei pietas infra septa hujus ecclesie sepeliri permiserit, omni anno ad sepulturam meam convenientes ...

${ }^{15}$ Acte de l'abbé Adam (Cartulaire blanc, t. I, p. $227=$ p. 35 de l'Ancien inventaire noir).

${ }^{16}$ La charte de Suger, après avoir évoqué l'évêché de Soissons en tête, parlait ici de mensura urbis. 
Retour sur le Cartulaire blanc de Saint-Denis

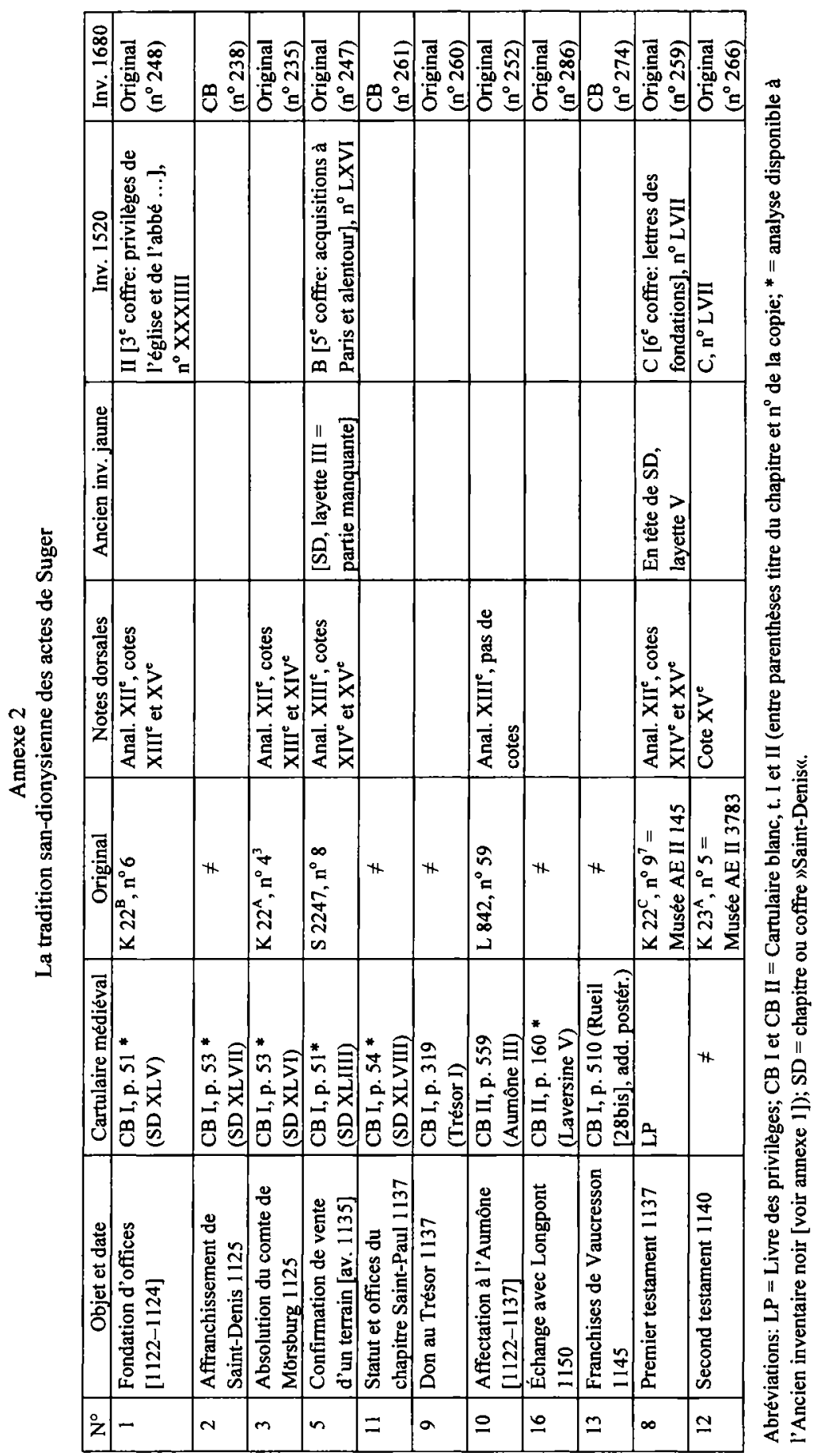


